\title{
SimBa: A Simulation and Balancing System for Manual Production Lines
}

\author{
Isabel C. Praça, Adriano S. Carvalho \\ Faculdade de Engenharia da Universidade do Porto \\ Instituto de Sistemas e Robótica - Grupo de Automação Industrial \\ R. dos Bragas 4099 Porto Codex - Portugal \\ Tel:02-2041619email isp@tormentas.fe.up.pt,asc@garfield.fe.up.pt
}

\begin{abstract}
The balancing and the comparison between different solutions through the simulation of operation of man based production lines is an important tool to help to decide properly at the level of production planning. This tool becomes much more important in the planning of lines based on variable performance work stations, due to needs of learning, fast changes of operations, which cause frequent bottlenecks and difficulties in previewing the future performance of the production lines.

In the paper the authors analyse this problem and present a validated solution to perform the line balance and to provide a tool to simulate posterior production to help the production manager configure the line according to the objective of balancing it.
\end{abstract}

\section{Keywords \\ flow lines, balancing of manual flow lines, simulation, manufacturing systems}

\section{INTRODUCTION}

The production planning is performed based on decisions on the shop floor at different levels. In manual manufacturing systems the production lines balancing becomes an important matter. The balancing, an issue classifiable as NP-hard, presents a practical solution based essentially on the application of heuristic rules.

Some of the questions to give answer are:

- How are the manufacturing operations to be allocated to work stations?

- What should the cycle time (production rate) be?

- Should any work stations be paralleled, i.e. duplicated, and should multiple manning be employed?

The dependency on the human performance causes great complexity in the analysis of manual manufacturing lines. In fact, taking into account standard times for the operations times estimated during the product design step - would allow lines balancing that do not 
guarantee a balanced operation in run time. The strong dependency of human performance in the operation times for this type of lines, influenced by factors such as absenteeism, the learning capacity and predisposition, the physical and emotional conditions of the operators, presents an additional requirement for evaluation of different factors and its role in the production planning.

In this context, simulation of the lines operation seems to become the appropriate tool for analysis and comparison of different line configurations according to predefined criteria. The simulation provides quantitative and qualitative information to the planner that allows him to take the right decision. So, it becomes an useful contribution to the planning system.

The tool presented in this paper was developed concerning the requirements of a production system organized as a set of manual flow lines. There was studied a set of three heuristic rules that allow the production manager to get different line balances in order to simulate them and evaluate their performance according to well defined and meaningful parameters.

This paper presents the characteristics and functionalities of a system, called SimBa, developed to balance and simulate manual production lines, according to appropriate rules. Some discussion on the role of simulation tools to help the production manager to take planning decisions is also presented.

\section{MANUAL FLOW LINES BALANCING AND SIMULATION}

The problem of balancing lines is a very important and complex one. Since the 50 decade several techniques and procedures have been developed, each having its own advantages and disadvantages. Those methods can be divided into optimal and heuristic procedures.

Optimal procedures require integer and linear programming algorithms and branch-andbound algorithms. In (Talbot, 1984), (Held, 1962), and (Bowman, 1959), some of them are described.

The line balancing is an NP-hard problem. That means that the computacional power to obtain an optimal solution increases exponentially with the increase in problem dimensions. Therefore, for large problems it is usual to employ heuristic methods capable of achieving good solutions in shorter time. The heuristic procedures can also be easily adapted to the caracteristics of each problem, such as restrictions on the number of components to be worked in each work station, mutually exclusive operations, multiple objectives and variable operation durations.

Several criterion can be used to compare different line configurations. The most important are:

- number of work stations (number of equipment and operators) needed;

- balancing loss;

- levels of stocks between work stations;

- bottleneck stations;

- work stations utilization;

- number of days to complete the production of all items.

The last four criteria can only be evaluated by means of simulating each line configuration in analysis, so it is noticeable the importance of simulation in this field.

Simulation is a technique used in the analysis of complex systems in which it is too expensive, difficult or even impossible to do experiences with the real system. Some of the 
application field of simulation is the study of production and economic systems, distribution networks, and scheduling of services and personnel.

Simulation is used essentially to acquire knowledge about a system and to do experiences that can help to evaluate future performance and evolution. The information provided by a simulation study becomes valuable in the support of decision making.

The simulation of manufacturing systems is frequently a discrete event simulation. So it is the simulation of flow lines, since what is relevant is the changes in system state after occurrence of well defined events. Some of the most relevant events during line operation are:

- the end of batch processing in a work station;

- the need of a new work station by a batch to be processed on;

- breakdown of equipment;

- lack of raw materials or components;

- absence of operator.

The possibility of using information provided by a system capable of obtaining and analysing different line configurations, allows the production manager to take better decisions.

\section{SIMBA SYSTEM}

The analysis of a production system organized as a set of several production lines and of the difficulties that the production manager has to deal with was the starting point for the development of this system.

It implements three heuristic rules, that allows the user to obtain different line configurations. These different line configurations can be compared by means of a simulation module which gives the user qualitative and quantitative information about the future performance of the system.

The general architecture of the SimBa system is described in the next figure.

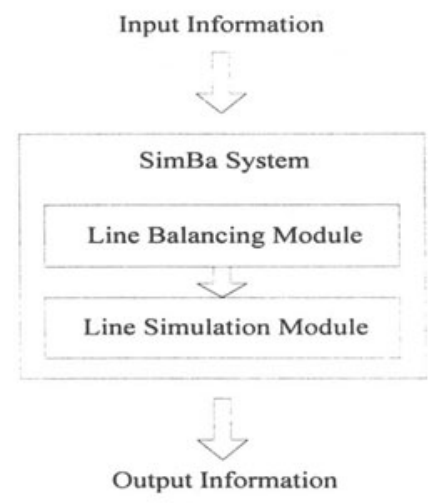

Figure 1 Architecture of SimBa system.

The system input information is related to:

- number, duration and operations precedence;

- number of items to produce; 
- learning curves of the operators;

- desired cycle time.

The SimBa system is constituted of two modules: the line balancing module and the line simulation module.

\subsection{Line Balancing Module}

The Line Balancing Module implements three heuristic rules which allow the user to obtain different line configurations. The criteria used, computational time involved in and probability of solving optimal solution, to select the heuristic rules were based on the performance of the rules in a comparative study developed by Boctor (Boctor, 1995). Boctor evaluates the performance of 16 heuristic rules in 67 problems taken from the open literature and in 700 randomly generated problems. The good performance of the rules, evaluated in the mentioned study before, assures they are capable of finding good solutions.

It must be taken into account the simplicity of the rules, since their simplicity allows to obtain fast results, an important factor when considering future improvements on the rules to adapt them to fit each particular case.

The selected rules were the Largest Candidate Rule, the Ranked Positional Weight Method and the Multiple Heuristic Rule.

\section{Largest Candidate Rule}

Using the Largest Candidate Rule, the operations are allocated to stations, beginning with the first station, by selecting whose operations are feasible in descending order of size. It is a very simple rule, future alterations can be easily made, and has proved good performance on the vast study realized by Boctor.

\section{Ranked Positional Weights Method}

With the Ranked Positional Weights Method it is necessary to find the positional weight of each operation. It is calculated by summing the operation's own standard time and the standard time for all following operations. The positional weight is, therefore, a measure of the operation time and its position in the sequence of operations. The operations are allocated to work stations in order of decreasing positional weight and without violating precedence constraints. It is more complex than the Largest Candidate Rule, however it is a simple rule that proved good performance on the mentioned study before.

\section{Multiple Heuristic Rule}

The Multiple Heuristic Rule was proposed by Boctor. It is a composite line balancing method were the operations are assigned to work stations in decreasing order of its priority. The priority of the operations is determined by applying several rules to the set of schedulable operations (operations for which all predecessors are already assigned to a work station). To choose among the schedulable operations, the one being assigned to the current work station select:

1. the operation having a duration equal to the remaining time.

2. the severe operation (operation having a processing time greater than or equal to one half of the cycle time) having the largest number of subsequent candidates (number of operations that become schedulable after assigning the operation in question to the work station). 
3. the combination of two operations having a duration equal to the remaining time.

4. the operation having the largest number of subsequent candidates.

To break ties in the four mentioned rules there are other defined rules.

The Multiple Heuristic Rule is the more complex of the three heuristic rules selected, however it was the one that prove better performance in the referred comparative study.

The information provided by the Line Balancing Module of the SimBa system is the number and the constitution of the work stations to be used in the production of the items. The user can obtain a line configuration for each of the three heuristic rules implemented, which can after be simulated in order to evaluate the future performance of the system and compare the solutions obtained.

\subsection{Line Simulation Module}

The simulation module allows the comparison of different line balances obtained by means of the heuristic rules implemented, or another suggested by the user.

It is possible to simulate the operating of lines with standard durations of the operations and also taking into account the learning curves of operators, which allows an analysis that reflects the variability of human performance. Using a simulation considering the learning curves of the operators allows the user to test different operators allocation in order to optimize the mapping between work places and operators.

It is a discrete event simulation based on a queuing model, where each work station is represented as having one or more servers and an associated waiting queue that represents the input buffer. The events are:

- Kickoff: start the production of the items, which can be based on batches, i.e. to start the production of the first batch or item at the work station where the first operation is assigned.

- Release_workstation: when an item or batch finishes processing in a work station.

- Request_workstation: when an item or batch request a work station in order to continue its production. If the next work station is occupied, the batch has to wait in the input buffer, otherwise it starts to be produced in it.

- NewBatch_type: when an assembly operation occurs it originates a new type of item.

- Update_operation_times: due to the learning curves of the operators the processing time of each operation will change along the time. This event deals with the necessity of updating the processing times.

The Line Simulation Module provides information about:

- levels of stocks between work stations;

- work stations utilization times;

- number of days to complete the production of all the items.

With this information it is possible to compare different line configurations. A line configuration with lower levels of stocks between the work stations, high utilization times and fewer days to complete the production of all the items is the most suitable.

The information concerning the levels of stocks in the input buffer of each work station allows the detection of bottleneck stations, which can be detected by the high level of stock in the input buffer and high utilization time.

Based on the output information of the SimBa system, the user can support the decision of choosing the line configuration that better fits the production. 
The SimBa system was developed according to an object oriented methodology, specifically the "Object Modelling Technique" by Rumbaugh (Rumbaugh, 1991). It was developed in $\mathrm{C}++$, being the Line Simulation Module also supported by Sim++ libraries of the SimPack package developed by Prof. Fishwick team at the University of Florida (Fishwick, 1992).

\section{EXAMPLE OF APPLICATION OF THE SIMBA SYSTEM}

In this section an example of the application of this system is described, in order to illustrate how it can help the managers for the production lines in taking decisions about the line configuration to implement.

The example shown is taken from a case study of a real production system organized as a manual production line. This and other examples can be found in (Praça, 1996).

The graph with the precedence restrictions of the operations necessary to produce one type of product made in that industry, as well as the operations durations, is shown in Figure 2.

\section{Input information}

The input information should be presented in the format:

Product Code 3830

Number of batches 50

Number of products in each batch 100

Number of operations 18

Number of branches of the operations graph 3

Cycle time 1.70

Operations:

code; duration; next operation; number of following operations; precedent operations; branch; initial $=1$

$$
\begin{aligned}
& 0->0.151001 \\
& 1->0.4021000 \\
& 2->0.5031100 \\
& \text { 3->0.1541200 } \\
& \text { 4->0.8051300 } \\
& \text { 5->0.2561400 }
\end{aligned}
$$

$$
\begin{aligned}
& \text { 6->0.20101500 } \\
& 7->0.658011 \\
& 8->0.3091710 \\
& 9->0.40101810 \\
& 10->1.701126920 \\
& 11->1.651211020
\end{aligned}
$$

$$
\begin{aligned}
& 12->0.4513111120 \\
& 13->0.35141112220 \\
& 14->0.9515111320 \\
& 15->0.1516114220 \\
& 16->0.45171115220 \\
& 17->0.90-11116220
\end{aligned}
$$

With this information the user can select which of the heuristic rules to use to obtain a line balance. It is possible to obtain three different line configurations. For each line configuration the results obtained with the Line Balancing Module show the constitution of the line, i.e. the number of work stations and the operations that were assigned to each one. 


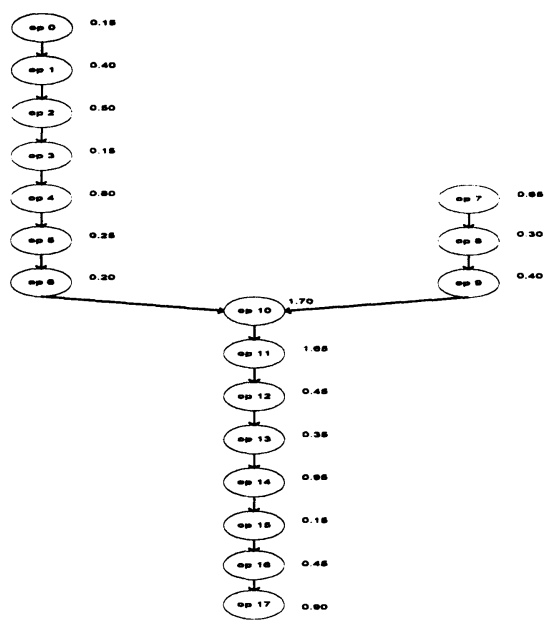

Figure 2 Graph of operations preference.

\subsection{Largest Candidate Rule}

Line configuration:

Number of work stations: 8

Work station: 1

Number of operations: 4

Operation: 7

Operation: 8

Operation: 9

Operation: 0

Work station: 2

Number of operations: 3

Operation: 1

Operation: 2

Operation: 3
Operation: 3

Work station: 3

Number of operations: 3

Operation: 4

Operation: 5

Operation: 6

Work station: 4

Number of operations: 1

Operation: 10

Work station: 5

Number of operations: 1

Operation: 11
Work station: 6

Number of operations: 2

Operation: 12

Operation: 13

Work station: 7

Number of operations: 3

Operation: 14

Operation: 15

Operation: 16

Work station: 8

Number of operations: 1

Operation: 17

For this and other line configurations, obtained by the Line Balancing Module or suggested by the user, the line can be simulated with standard duration of the operations or taking into account the variability introduced by the operators learning curve.

In this example the results obtained in both cases are illustrated.

\section{Results obtained with the standard durations:}

Days to produce all items: 18 
System Utilization: $70.4 \%$

Arrival Rate: 0.010828 , Throughput: 0.005414

Work stations Statistics

F 1 (work station): Idle: $18.8 \%$, Util: $81.2 \%$, Preemptions: 0, Longest Q: 2 F 2 (work station): Idle: $43.2 \%$, Util: $56.8 \%$, Preemptions: 0, Longest Q: 0 F 3 (work station): Idle: $32.3 \%$, Util: $67.7 \%$, Preemptions: 0, Longest Q: 0 F 4 (work station): Idle: $8.0 \%$, Util: $92.0 \%$, Preemptions: 0, Longest Q: 6 F 5 (work station): Idle: $10.7 \%$, Util: $89.3 \%$, Preemptions: 0, Longest Q: 0 F 6 (work station): Idle: $56.7 \%$, Util: $43.3 \%$, Preemptions: 0, Longest Q: 0 F 7 (work station): Idle: $16.1 \%$, Util: $83.9 \%$, Preemptions: 0, Longest Q: 0 F 8 (work station): Idle: $51.3 \%$, Util: $48.7 \%$, Preemptions: 0, Longest Q: 0

\section{Results considering the learning curves of the operators:}

Days to produce all items: 15

System Utilization: $71.2 \%$

Arrival Rate: 0.013508 , Throughput: 0.006754

Work station Statistics

F 1 (work station): Idle: $19.0 \%$, Util: $81.0 \%$, Preemptions: 0, Longest Q: 2 F 2 (work station): Idle: $44.4 \%$, Util: $55.6 \%$, Preemptions: 0, Longest Q: 0 F 3 (work station): Idle: $35.0 \%$, Util: $65.0 \%$, Preemptions: 0, Longest Q: 0 F 4 (work station): Idle: $8.1 \%$, Util: $91.9 \%$, Preemptions: 0, Longest Q: 6 F 5 (work station): Idle: $16.4 \%$, Util: $83.6 \%$, Preemptions: 0, Longest Q: 0 F 6 (work station): Idle: $48.4 \%$, Util: $51.6 \%$, Preemptions: 0, Longest Q: 0 F 7 (work station): Idle: $17.4 \%$, Util: $82.6 \%$, Preemptions: 0, Longest Q: 0 F 8 (work station): Idle: $41.7 \%$, Util: $58.3 \%$, Preemptions: 0, Longest Q: 0

\subsection{Ranked Positional Weight Method}

Line configuration obtained through the Ranked Positional Weight Method:

Number of work stations: 8

$\begin{array}{lll}\text { Work station: } 1 & \text { Work station: } 3 & \text { Work station: } 6 \\ \text { Number of operations: } 4 & \text { Number of operations: } 3 & \text { Number of operations: } 2 \\ \text { Operation: } 0 & \text { Operation: } 8 & \text { Operation: } 12 \\ \text { Operation: } 1 & \text { Operation: } 9 & \text { Operation: } 13 \\ \text { Operation: } 2 & \text { Operation: } 6 & \text { Work station: } 7 \\ \text { Operation: } 3 & \text { Work station: } 4 & \text { Number of operations: } 3 \\ \text { Work station: } 2 & \text { Number of operations: } 1 & \text { Operation: } 14 \\ \text { Number of operations: } 3 & \text { Operation: } 10 & \text { Operation: } 15 \\ \text { Operation: } 7 & \text { Work station: } 5 & \text { Operation: } 16 \\ \text { Operation: } 4 & \text { Number of operations: } 1 & \text { Work station: } 8 \\ \text { Operation: } 5 & \text { Operation: } 11 & \text { Number of operations: } 1 \\ & & \text { Operation: } 17\end{array}$




\section{Results obtained with the standard durations:}

Days to produce all items:24

System Utilization: $52.5 \%$

Arrival Rate: 0.008078 , Throughput: 0.004039

Work station Statistics

F 1 (work station): Idle: $51.5 \%$, Util: $48.5 \%$, Preemptions: 0, Longest Q: 1 F 2 (work station): Idle: $31.3 \%$, Util: $68.7 \%$, Preemptions: 0, Longest Q: 5 F 3 (work station): Idle: $63.7 \%$, Util: $36.3 \%$, Preemptions: 0, Longest Q: 3 F 4 (work station): Idle: $31.3 \%$, Util: $68.7 \%$, Preemptions: 0, Longest Q: 18 F 5 (work station): Idle: $33.4 \%$, Util: $66.6 \%$, Preemptions: 0, Longest Q: 0 F 6 (work station): Idle: $67.7 \%$, Util: $32.3 \%$, Preemptions: 0, Longest Q: 0 F 7 (work station): Idle: $37.4 \%$, Util: $62.6 \%$, Preemptions: 0, Longest Q: 0 F 8 (work station): Idle: $63.7 \%$, Util: $36.3 \%$, Preemptions: 0, Longest Q: 0

\section{Results considering the learning curves of the operators:}

Days to produce all items: 19

System Utilization: $54.9 \%$

Arrival Rate: 0.010369 , Throughput: 0.005184

Work station Statistics

F 1 (work station): Idle: $50.2 \%$, Util: $49.8 \%$, Preemptions: 0, Longest Q: 1 F 2 (work station): Idle: $30.8 \%$, Util: 69.2\%, Preemptions: 0, Longest Q: 4 F 3 (work station): Idle: $63.8 \%$, Util: $36.2 \%$, Preemptions: 0, Longest Q: 2 F 4 (work station): Idle: $29.5 \%$, Util: $70.5 \%$, Preemptions: 0, Longest Q: 17 F 5 (work station): Idle: $34.9 \%$, Util: $65.1 \%$, Preemptions: 0, Longest Q: 0 F 6 (work station): Idle: $60.0 \%$, Util: $40.0 \%$, Preemptions: 0, Longest Q: 0 F 7 (work station): Idle: $36.4 \%$, Util: $63.6 \%$, Preemptions: 0, Longest Q: 0 F 8 (work station): Idle: $54.9 \%$, Util: $45.1 \%$, Preemptions: 0, Longest Q: 0

\section{Discussion of the results}

With the information provided by the Line Balancing Module of the SimBa system it becomes possible to compare different line configurations which gives support to the decision of choosing the best one to implement in the production of the items.

In the example given, both configurations have the same number of work stations, and so the quantity of human and material resources necessary to the production will not affect the choice between them.

In the line configuration obtained through the Ranked Positional Weight Method the work station 4 has a huge accumulation of items in the input buffer. It is probably a bottleneck station, so the manager must take this into account in the decision making. Also, the number of days to complete the work is less with the configuration obtained with the Largest Candidate Rule and the levels of stocks are shorter. These factors and the higher utilization times of the work stations suggest the performance of this line configuration will be better. 
But, the user can also compare it with other solutions and do some changes in order to find a better one.

The SimBa application in the real context has validated the results showed, through comparison with lines performance obtained by the methods used. In (Praça, 1996) the system is described with enough detail and more examples of its application to the real system are given and discussed.

\section{CONCLUSIONS}

This paper presents an approach to achieve good line balances, namely lines that involve a great component of manual work.

Simulation is an important framework in the analysis of flows global performance, and in the evaluation of alternative configurations. With simulation it is possible to obtain information on the performance evolution of lines, namely the detection of bottleneck stations and high levels of stocks.

So the use of simulation in a system capable of giving the user quantitative and qualitative information about alternative line configurations helps the production manager to compare different solutions, and so helps him to make decisions.

With SimBa, a developed and presented manufacturing application, becomes possible to obtain different line balances based on different heuristic rules implemented. The evaluation of this balances through simulation is very important in order to take correct decisions. The well performed detection of high levels of stocks, and so of bottlenecks, is a strong advantage of this system.

With this system different operator distributions can be tested, in order to achieve the best solution. It is possible then to establish a correct allocation of operators to the work stations, based on the results obtained when considering their learning curves during the simulation.

\section{REFERENCES}

Boctor, Fayez F.(1995) A Multiple-rule Heuristic for Assembly Line Balancing. Journal of the Operational Research Society

Bowman, E. H. (1959) Assembly-Line Balancing by Linear Programming. Massachusetts Institute of Technology, Cambridge

Fishwick, Paul A.(1992) Simpack: Getting Started width Simulation Programming In C And C++. Dept. of Computer \& Information Science, University of Florida

Held, Michael, Karp, Richard M. and Shareshian, Richard (1962) Assembly-Line BalancingDynamic Programming with Precedence Constraints. International Business Machines Corporation, New York"

Praça, Isabel C. (1996) Simulação e Balanceamento de Linhas Manuais de Fabrico. Masters Thesis. Faculdade de Engenharia da Universidade do Porto.

Rumbaugh, James(1991) Object-Oriented Modelling and Design. Prentice-Hall Internacional Talbot, F. Brian and Patterson, James H. (1984) An Integer Programming Algorithm with Network Cuts for Solving the Assembly Line Balancing Problem. Management Science vol. 30 